\title{
A Geometry-Inclusive Analysis for Single-Relay Systems
}

\author{
Meng Yu, ${ }^{1}$ Jing (Tiffany) Li, ${ }^{1}$ and Hamid Sadjadpour ${ }^{2}$ \\ ${ }^{1}$ Department of Electrical and Computer Engineering, Lehigh University, Bethlehem, PA 18015, USA \\ ${ }^{2}$ Baskin School of Engineering, University of California, Santa Cruz, CA 95064, USA
}

Correspondence should be addressed to Meng Yu, mey3@lehigh.edu

Received 14 January 2009; Accepted 31 March 2009

Recommended by Daniel Iancu

Successful message relay, or the quality of the interuser channel, is critical to fully realize the cooperative benefits promised by the theory. This in turn points out the importance of the geometry of cooperative system. This paper investigates the impact of the relay's location on the system capacity and outage probability for both amplify-forward (AF) and decode-forward (DF) schemes. Signal attenuation is modeled using power laws, and capacity is evaluated using the max-flow min-cut theory. A capacity contour for DF, the more popular mode of the two, is provided to facilitate the derivation of engineering rules. Finally, a selective singlerelay system, which selects the best relay node among a host of candidates according to their locations, is analyzed. The average system capacity and outage, averaged over all possible candidates' locations, are evaluated. The result shows that the availability of a small candidate pool of 3 to 5 nodes suffices to reap most of the cooperative gains promised by a selective single-relay system.

Copyright () 2009 Meng Yu et al. This is an open access article distributed under the Creative Commons Attribution License, which permits unrestricted use, distribution, and reproduction in any medium, provided the original work is properly cited.

\section{Introduction}

Aside from temporal and frequency diversities, spatial diversity is another technique to mitigate the deterioration caused by fading. Due to the limitation on the size of mobile terminals, multiple antennas are not always practical. As a remedy to this, user cooperation has been proposed [1, 2 ], where multiple users share antennas to form a virtual antenna array and obtain spatial diversity.

Aiming at increasing the channel capacity or decreasing the outage probability or both, several interesting cooperative protocols have been proposed (e.g., [2, 3]). Among them, amplify-forward (AF) and decode-forward (DF) are the two fundamental forwarding modes. Their qualities have been studied by many researchers both from the information theoretic aspects and the practical aspects (e.g., [2, 4]). Reference [5] evaluated their performances in practical wireless scenarios in general, and the interuser outage case in particular. (By an interuser outage, we mean that the relay is unable to extract a clean copy of the source data.) It was shown in [5] that (1) the interuser outage happens at a nonnegligible probability even with decent channel code protection; for example, on block Rayleigh fading channels with an interuser signal-to-noise ratio (SNR) of $10-22 \mathrm{~dB}$, the interuser outage happens at a probability of
$10.4 \%-1.06 \%$ even with the protection of a $(3000,2000)$ random low-density parity-check (LDPC) code; (2) when an interuser outage happens, both AF and DF perform badly with an effective diversity order of only 1 ; (3) the overall system performance is to a large extend limited by this worstcase scenario. These results revealed that a high-quality interuser channel is one key to realize the great benefits that user cooperation may offer.

Prior results in turn point out the importance of the location of the relay. On one hand, if the system cannot choose its relay's location, what is the average performance of a cooperative system when the relay can move to any place (bad location or good location) in a region around the source and the destination? Or how much does a relay node picked up randomly help the system? On the other hand, if the system can judiciously choose its relay partner among a host of candidate nodes, what would be the desired location for the relay? How much benefits if many candidate nodes are available?

Intuitively, when the relay gets close to the source, the interuser outage tends to diminish and the cooperative system tends to resemble a 2-by-1 multiple-input singleoutput (MISO) system, but how close is close? Further, because 2-by-1 and 1-by-2 systems are capacity comparable, does this suggest that a relay close to the destination would 
also work in favor? More generally, is there a symmetry or duality property in the relay system?

The purpose of this paper is to answer the above questions and to understand the effect caused by the location of the relay. Reference [6] analyzed the performance of relay networks based on achievable rate region. This paper focuses on capacity evaluation, including the ergodic channel capacity and the outage probability. Capacity by definition establishes limits on the performance of practical communication systems. These limits provide system benchmarks and reveal how much improvement is theoretically possible. Several researchers have studied the information-theoretic aspects of the two-transmitter one-destination wireless cooperative system but only for a few samples of fixed channel qualities. In this work, we also study the capacity as a function of geometry. A similar study was conducted for the Gaussian channels in [7]. We consider both DF and AF modes for the single-relay cooperative system on Rayleigh fading channels using power law air propagation models. The system limits are first analyzed using the max-flow min-cut theory for different relay locations in several topologies. The results show that, in $\mathrm{AF}$, system achieves high performance when the relay is near the median line between the source and the destination in most cases; in DF, system achieves high capacity when the relay is at the source side. It is worth mentioning that our analysis reveals a symmetry property in the capacity for AF, but not in DF. The reason behind it, particularly in terms of why DF does not mimic multipleinput multiple-output (MIMO) systems, is provided. A capacity contour for DF, the more practical and useful mode of the two, is subsequently computed. The capacity contour clearly provides motivation and guidelines for choosing good partners in practical situations. Following these results, a selective single-relay system, which selects the best relay node among a host of candidate nodes, is analyzed and evaluated. Different from [8], we consider effects of the number of potential relays for a single-relay system and use ergodic capacity or outage probability as selection criteria. We consider uniform random distributions of the candidates and compute the average system capacity and outage, where the average is taken among all possible candidates' locations. The results show that when the signal attenuation due to path loss is severe, or when there are at least two candidates available (so that the better one is selected as the relay), the gain achieved through user cooperation always outweighs the rate loss due to relaying. Further, it is sufficient to keep track of only a small pool of 3-5 candidates to reap most of the gain available to a single-relay system.

In the body of this paper, Section 2 briefs on the system model and cooperative modes. Section 3 analyzes the ergodic capacity and outage probability affected by the relay at different locations, and gives a capacity contour. Section 4 briefs the selective single-relay system and analyzes its performance. Section 5 presents our conclusion.

\section{User Cooperation}

2.1. System Model. In this paper we consider single-relay wireless system. Let "home channel", "interuser channel", and "relay channel" denote the channels between the source and the destination, the source and the relay, and the relay and the destination, respectively. Let $h_{S D}, h_{S R}$, and $h_{R D}$ denote the respective channel gains. Since user cooperation is most useful when channels are varying very slowly (i.e., hard to obtain time diversity in a single user channel), the channels are modeled as Rayleigh block fading channels, where channels remain constant for the duration of one round of user cooperation (2 consecutive time slots). Between channels and cooperation rounds, the channel gains are independent. The general form of a signal received over a specific channel at time $t$ is given by

$$
y(t)=\sqrt{E_{s}} h(t) x(t)+n(t),
$$

where $E_{s}$ is the signal energy, $h(t)$ is the channel gain, and $n(t)$ is the additive white Gaussian noise (AWGN) with a power spectrum density $N_{0}$. The channel gain follows

$$
h(t)=\alpha \phi(t)
$$

where channel fading coefficient $\phi(t)$ is a random variable that follows the Rayleigh distribution, and $\alpha$ is the pathloss. We assume that the square of the pathloss is inversely proportional to some power of the distance, that is, $\alpha^{2}=$ $l^{-\delta}$, where $l$ is the distance between the transmitter and the receiver, and $\delta$, an integer between $[2,4]$, is the pathloss exponent. (To ease the evaluation, we consider isotropic signal propagation model within each topological setup. In reality, however, the propagation model also depends on the environment and the transmission distance.)

Among the various possible strategies of user cooperation (e.g., [2, 3]), we consider half-duplex systems, the simplest type, where after the source transmits a package in the first time slot, the relay forwards the message in the second time slot. We assume that the channel side information (i.e., channel gain) is known to the respective receivers, and the power is equally allocated in the two time slots.

2.2. Fundamental Cooperative Modes. At the first time slot, the signal received at the destination is

$$
y_{D, 1}=\sqrt{E_{s}} h_{S D} x_{1}+n_{0}
$$

where $n_{0}$ denotes the zero-mean complex AWGN.

2.2.1. AF Mode. We assume that the power of the signal retransmitted at the relay node is scaled uniformly with respect to all the bits in the package, such that the average (re)transmission energy per signal equals $E_{s}$.

In the second time slot, the signal received at the destination is

$$
y_{D, 2}^{\mathrm{AF}}=h_{R D}\left|h_{S R}\right| \sqrt{\frac{E_{s}^{2}}{E_{s}\left|h_{S R}\right|^{2}+N_{0}}} x_{1}+\tilde{n},
$$

where $\tilde{n}$ is a zero mean complex Gaussian noise with a variance of $\left(N_{0} / 2+N_{0}\left|h_{R D}\right|^{2} E_{s} / 2\left(E_{S}\left|h_{S R}\right|^{2}+N_{0}\right)\right)$ per 
dimension [4]. The destination combines $y_{D, 1}$ and $y_{D, 2}^{\mathrm{AF}}$ using the maximal ratio combination (MRC) rule before decoding.

Let

$$
C(\gamma) \triangleq \frac{1}{2} \log _{2}(1+\gamma) \mathrm{bit} / \mathrm{s} / \mathrm{Hz}
$$

denote the capacity of a single Gaussian channel in a cooperative system with a signal-to-noise ratio $\gamma$. The factor $1 / 2$ is introduced to account for the fact that two consecutive time slots are used for each package.

For AF, it is easy to see that the achievable (instantaneous) information rate is upper bounded by the (instantaneous) mutual information of the compound channel [2]:

$$
R^{\mathrm{AF}} \leq I^{\mathrm{AF}}=C\left(\left\|\gamma^{\mathrm{AF}}\right\|_{1}\right)
$$

where

$$
\begin{gathered}
\gamma^{\mathrm{AF}}=[\underbrace{\frac{E_{s}\left|h_{S D}\right|^{2}}{N_{0}}}_{\text {1st time slot }}, \underbrace{\frac{E_{s}^{2}\left|h_{R D} h_{S R}\right|^{2}}{\left(E_{s}\left|h_{R D}\right|^{2}+E_{s}\left|h_{S R}\right|^{2}+N_{0}\right) N_{0}}}_{\text {2nd time slot }}], \\
\|\mathbf{a}\|_{1}=\sum_{i}\left|a_{i}\right| .
\end{gathered}
$$

That $h_{S R}$ and $h_{R D}$ are interchangeable in the above SNR formulation suggests a capacity symmetry with respect to the position of the source and the destination in AF.

2.2.2. DF Mode. In DF, the relay demodulates and decodes the packet and forwards part or all of the information possibly using a different (compression or error control) code. We note that the decode-forward strategy we consider here has certain flavor of the compression-forward (CF) mode discussed in [9]. The difference, however, is that the relay in CF needs not to decode the message and, rather, forwards compressed versions of its observations. With different channel states, different coding strategies may outperform each other $[10,11]$. We assume that the system can switch between coding strategies to exploit the channel capacity. From network information theory, one realizes that the achievable information rate of DF is determined by the max-flow min-cut of the system. The cut set around the source forms a broadcast channel, and the cut set around the destination forms a parallel channel (because of the orthogonality in time). The system capacity is the minimum value of the two cut sets' capacity. Within each time slot, the channels can be treated as Gaussian channels. Because a Gaussian broadcast channel is a degraded broadcast channel, the better channel can always carry the information intended for the worse channel also. The capacity of the broadcast cut set is therefore the one with better signal-to-noise ratio:

$$
C_{\text {cut } 1}=\max \left\{C_{S D}, C_{S R}\right\},
$$

where

$$
\begin{aligned}
& C_{S D} \triangleq C\left(\frac{E_{s}}{N_{0}}\left|h_{S D}\right|^{2}\right), \\
& C_{S R} \triangleq C\left(\frac{E_{s}}{N_{0}}\left|h_{S R}\right|^{2}\right) .
\end{aligned}
$$

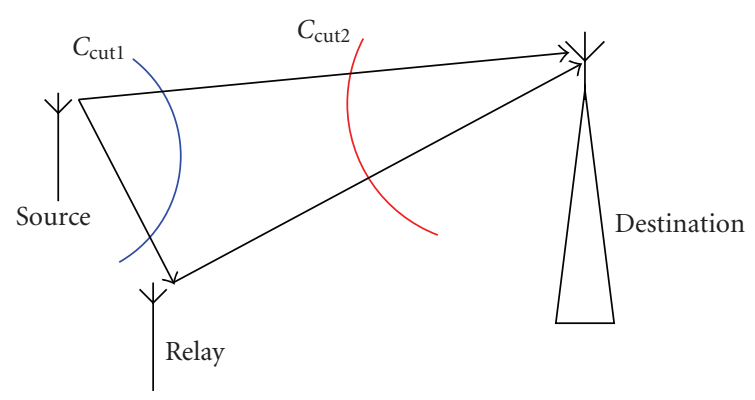

Figure 1: Cut sets of relay system.

On the other hand, the capacity of the cut set around the destination is the sum rate of the two paralleled channels:

$$
\begin{aligned}
& C_{\mathrm{cut} 2}=C_{S D}+C_{R D}, \\
& C_{R D} \triangleq C\left(\frac{E_{s}}{N_{0}}\left|h_{R D}\right|^{2}\right) .
\end{aligned}
$$

Hence, the system's instantaneous achievable rate is

$$
R^{\mathrm{DF}} \leq \min \left\{C_{\text {cut } 1}, C_{\text {cut } 2}\right\} .
$$

Based on the relative value of $C_{S D}, C_{S R}$, and $C_{R D}$, the detailed (instantaneous) information rate for DF is upper bounded by

$$
R^{\mathrm{DF}} \leq \begin{cases}C_{S D}, & \text { if } C_{S R} \leq C_{S D}, \\ C_{S R}, & \text { if } C_{S D}<C_{S R} \leq C_{S D}+C_{R D}, \\ C_{S D}+C_{R D}, & \text { if } C_{S D}+C_{R D}<C_{S R} .\end{cases}
$$

Now that we have the instantaneous rates for the $\mathrm{AF}$ and DF system in (6) and (13), respectively, we can average them over the distribution of the channel gain (a Rayleigh distribution whose mean is some power of the distance) to account for the signal attenuation caused by the channel fading and the geometry of the terminals. These results are plotted in Figures 3 and 6 and are discussed in the succeeding sections.

\section{Capacity and Outage at Different Locations}

3.1. Ergodic Capacity. Ergodic capacity, more commonly known as the Shannon limit, determines the maximum achievable information rate averaged over all fading states. Under the assumption that the system can adopt appropriate strategy to exploit the capacity, we have the ergodic capacity as

$$
\begin{aligned}
& C_{\mathrm{erg}}^{\mathrm{AF}}=\iiint R^{\mathrm{AF}} \mathrm{d} h_{S R} \mathrm{~d} h_{S D} \mathrm{~d} h_{R D}, \\
& C_{\mathrm{erg}}^{\mathrm{DF}}=\iiint R^{\mathrm{DF}} \mathrm{d} h_{S R} \mathrm{~d} h_{S D} \mathrm{~d} h_{R D} .
\end{aligned}
$$

As shown in Figure 2, the source and the destination are distributed horizontally with a distance $l_{S D}$, which is 


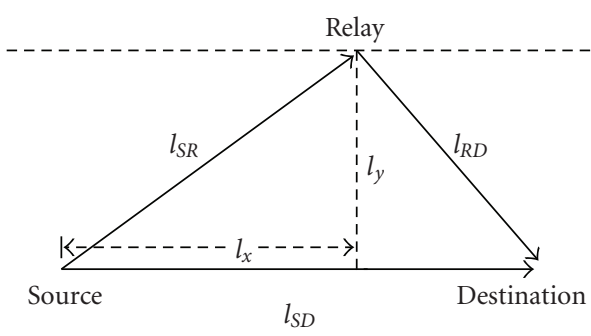

FIGURE 2: Geometry model.

normalized to unity. The horizontal distance and vertical distance from the relay to the source are $l_{x}$ and $l_{y}$. Let us begin evaluation with a fixed $l_{y}$ and a varying $l_{x}$ first.

Figure 3 shows the ergodic capacity where $l_{S D}=1, E_{s}=$ $1, N_{0}=1$, and $l_{y}=0.5$. Solid curves represent AF, dashed curves represent DF, and power law propagation models of $\delta=2,3,4$ are evaluated. From the curves, we can see that regardless of the value of $\delta$, the capacity of the AF system exhibits a symmetry property, and for the cases we tested, the maximum value is achieved at the median point. The former observation confirms that the positions of the source and the destination are interchangeable, as is implied in (7).

For the cases we tested, to analyze the effect of the relay's location, we take the model with $\delta=2$ as an example. First, note that the effective SNR of the AF system is the sum SNRs of two spatially independent channels: the direct channel between the source and the destination, and the cascade channel consisting of the source-relay channel and the relaydestination channel. The SNR of the former is irrespective to the relay, and the SNR of the latter varies with the location of the relay. The average SNR of the cascade channel is an integral over all states of $h_{S R}$ and $h_{R D}$. We analyze the value when $h_{S R}$ and $h_{R D}$ are around their mean values, which plays a major part in integral because of its high probability. At this time, the SNR of the cascade channel can be transformed into the following expression:

$$
\begin{aligned}
& \gamma_{\text {cas }} \\
& \propto \frac{1}{l_{S R}^{2}+l_{R D}^{2}+l_{S R}^{2} l_{R D}^{2}\left(N_{0} / E_{s}\right)} \\
& =\frac{1}{\left(N_{0} / E_{s}\right)\left(l_{S R}^{2}+E_{s} / N_{0}\right)\left(l_{R D}^{2}+E_{s} / N_{0}\right)-E_{s} / N_{0}} \\
& =\frac{1}{\left(N_{0} / E_{s}\right)\left(l_{x}^{2}+l_{y}^{2}+E_{s} / N_{0}\right)\left[\left(l_{S D}-l_{x}\right)^{2}+l_{y}^{2}+E_{s} / N_{0}\right]-E_{s} / N_{0}},
\end{aligned}
$$

where $l_{S D}, l_{R D}$, and $l_{S R}$ are the distances between the respective nodes. The main part of the denominator can be written as

$$
\begin{aligned}
\left(l_{x}^{2}+l_{y}^{2}+\frac{E_{s}}{N_{0}}\right)\left[\left(l_{S D}-l_{x}\right)^{2}+l_{y}^{2}+\frac{E_{s}}{N_{0}}\right] \\
\left.=\left(l_{x}^{2}+A^{2}\right)\left[\left(l_{S D}-l_{x}\right)^{2}+A^{2}\right)\right] \\
=l_{S D}^{2} A^{2}+\left[\frac{1}{4}\left(l_{S D}^{2}-4 A^{2}\right)-\left(\frac{1}{2} l_{S D}-l_{x}\right)^{2}\right]^{2},
\end{aligned}
$$

where $A^{2}=l_{y}^{2}+E_{s} / N_{0}$ is a constant value. If $l_{S D}^{2} \leq$ $4 A^{2}$, the denominator achieves its minimum when $l_{x}=$ $0.5 l_{S D}$. Otherwise, the denominator has two minimum values achieved when

$$
l_{x}=0.5 l_{S D} \pm \sqrt{0.25 l_{S D}^{2}-A^{2}} .
$$

Accordingly, the SNR of the cascade channel, and subsequently the effective SNR of the the AF system, will reach either one maximum value on the median line between the source and the destination or two maximum values located symmetrically on either side of the median line.

Figure 4 demonstrates an example of the latter case. A relatively low transmit power of $E_{s}=0.24$ is used, and both the ergodic capacity and the outage probability are plotted as functions of the relay location. It should be noted that the two optimal locations specified in (17) are for Gaussian channels. To evaluate fading channels, one needs to average over all the instantaneous Gaussian channel realizations (pertaining to the fading distribution). With a small transmit power such as $E_{s}=0.24$, the case of $l_{S D}^{2}>$ $4 A^{2}$ dominates; so there exit two optimal locations (which are functions of the fading coefficient) most of the time and one optimal location (at the median point). The best locations are selected by evaluating the average information rate over all the possible channel realizations. In terms of evaluating the outage probability, the curve may look slightly different with respect to different threshold values. In the Figure, the optimal locations obtained by the capacity and the outage results do not coincide exactly, but they are quite close. Unlike one may expect, these optimal locations are actually closer to the source and the destination than to their median position. We also observe that an arbitrary position between the two optimal locations suffers but only a small performance degradation. This suggests that the median position is nonetheless a convenient and safe choice for the relay.

The symmetry property, however, is not observable in DF. The capacity of DF peaks out when the relay sits at some position near the source, but unlike one would expect from a 2-by-1 MISO system, the optimal location is not very close to the source but appears to be between the $1: 9$ and $3: 7$ sections from the source to the destination for different propagation models. Recall that the capacity of DF is $\min \left\{C_{\text {cut } 1}, C_{\text {cut } 2}\right\}$. As the relay moves from the source to the destination, the increase of $C_{\text {cut1 }}$ will cause the decrease of $C_{\text {cut2 }}$ and vice versa. Hence, the maximum value is achieved when $C_{\text {cut1 }}=C_{\text {cut2 }}$. When $\delta$ is large, that is, high-order signal attenuation, $C_{S R}$ and $C_{R D}$ tend to dominate $C_{\text {cutl }}$ and $C_{\text {cut2 }}$, making the capacity curve closer to symmetric and the optimal relay location closer to the median line. Additionally, we see that each capacity curve consists of three segments. The first segment, spanning from the source to the optimal relay location, represents the case when the cut set around the destination $\left(C_{\text {cut } 2}\right)$ is the bottleneck for information flow. In that case, the capacity increases as the relay moves toward the destination. The second segment represents the case when the cut set around the source $\left(C_{\text {cut } 1}\right)$ dominates, 


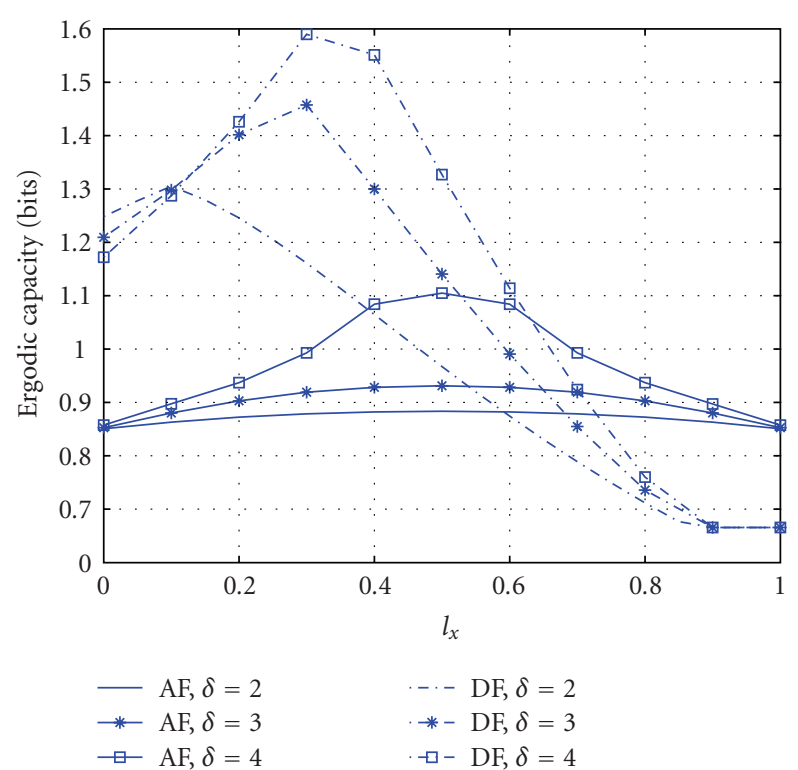

Figure 3: Ergodic capacity versus the location of the relay, $E_{s}=$ $1, N_{0}=1, l_{y}=0.5$, and $l_{S D}=1$.

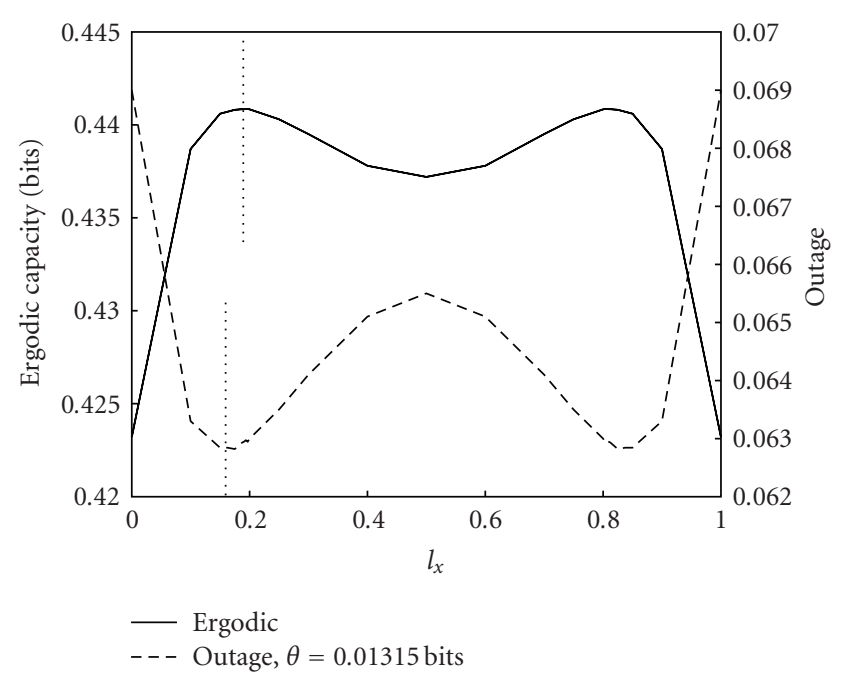

Figure 4: Ergodic capacity and outage $(\theta=0.01315)$ of AF versus the location of the relay, $\delta=2, E_{s}=0.24, N_{0}=1, l_{y}=0.05$, and $l_{S D}=1$.

and consequently the capacity decreases as the relay moves away from the source. Finally the capacity reaches a floor that is irrelevant to the relay location. This happens when the quality of the interuser channel is worse than the home channel, that is, $l_{S R}>l_{S D}$, and the relay system reverts to the noncooperative mode.

3.2. Outage Probability. Outage probability, aka outage capacity or simply, outage, is another important statistical measure for the quality of a fading channel especially in slow fading cases. Outage specifies the probability that the instantaneous channel quality fails to meet a satisfactory threshold $\theta$. Using information rate as the measure for channel quality, the outage probability for a single channel can be computed using

$$
P_{\text {out }}(\theta) \triangleq \operatorname{Pr}(R<\theta)=\int_{0}^{\theta} f_{C}(c) \mathrm{d} c,
$$

where $f_{C}(c)$ is the probability density function of the instantaneous information rate of that channel.

As shown in (7), the AF system can be viewed as a single channel with an effective SNR $\left\|\gamma^{\mathrm{AF}}\right\|_{1}$; hence, the outage probability can be evaluated numerically using (18).

For DF, the outage needs to be evaluated with respect to the cases when the cut set around the source or around the destination dominates. The former is a degraded broadcast channel where outage happens when $C_{\text {cut1 }}=$ $\max \left(C_{S R}, C_{S D}\right)<\theta$. The latter is a parallel channel where outage happens when $C_{\text {cut2 }}=C_{S D}+C_{R D}<\theta$. Overall the outage for DF system can be computed as

$$
\begin{aligned}
P_{\text {out }}= & 1-\operatorname{Pr}\left(C_{\text {cut } 1}>\theta\right) \operatorname{Pr}\left(C_{\text {cut } 2}>\theta\right) \\
= & \operatorname{Pr}\left(\max \left(C_{S R}, C_{S D}\right)<\theta\right) \\
& \cdot \operatorname{Pr}\left(\max \left(C_{S R}, C_{S D}\right)<C_{S D}+C_{R D}\right) \\
& +\operatorname{Pr}\left(C_{S D}+C_{R D}<\theta\right) \\
& \cdot \operatorname{Pr}\left(\max \left(C_{S R}, C_{S D}\right)>C_{S D}+C_{R D}\right) \\
= & \operatorname{Pr}\left(C_{S R}<\theta\right) \operatorname{Pr}\left(C_{S D}<\theta\right) \\
& +\left(1-\operatorname{Pr}\left(C_{S R}<\theta\right)\right) \operatorname{Pr}\left(C_{S D}+C_{R D}<\theta\right) .
\end{aligned}
$$

Figure 5 shows the outage probabilities of the model with a threshold $\theta=0.35$ bits per channel use, $N_{0}=0$, and $E_{s}=1$. The outage results appear quite consistent with the capacity results. For AF, the outage curve is also symmetric, and the lowest outage is achieved when the relay resides in equal distances between the source and the destination when the transmission power is high. For DF, the optimal relay position in terms of the least outage is somewhere between the $3: 7$ sections from the source to the destination.

Figure 4 is a case when the transmission power is low $\left(E_{s}=0.24\right)$. The dashed curve represents the outage with a threshold $\theta=0.01315$ bits. There are two symmetric optimal locations; however they are not the same ones of the ergodic capacity. Though not shown here, different threshold also has different optimal locations. This is because different instantaneous channel fades have the similar effects as different transmission powers, and different transmission powers may correspond to different optimal locations. The optimal locations for the outage threshold $\theta$ are averaged from 0 to $\theta$. This means that different criteria (ergodic capacity or outage, outages with different thresholds) may have different optimal locations.

When the transmission power is low, the optimal location is not on the median line (the numerical results are not shown here). 


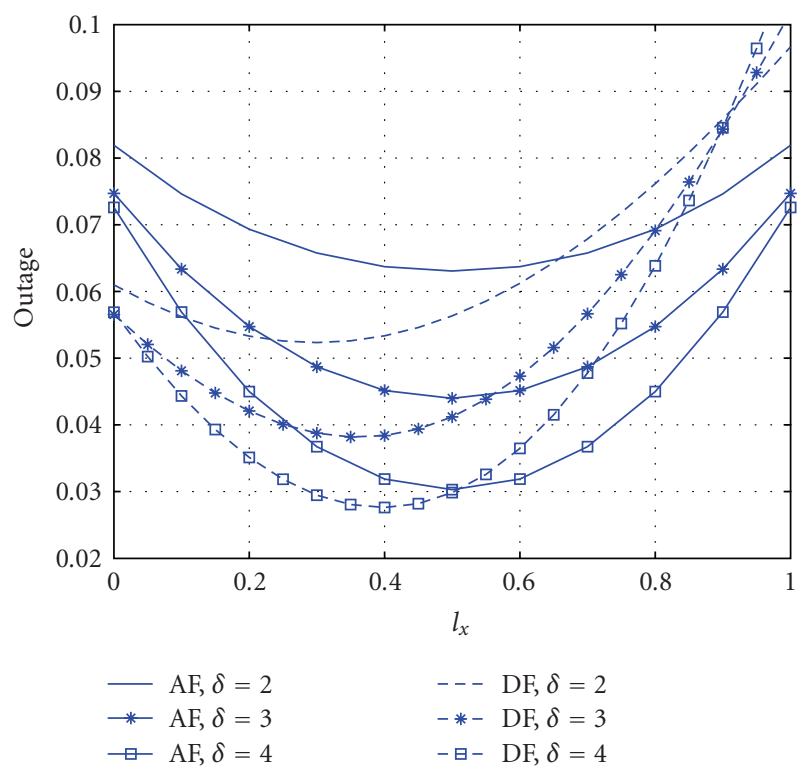

FIGURE 5: Outage probability versus the location of the relay, $E_{s}=1$, $N_{0}=1, y=0.5$, and $l_{S D}=1$.

3.3. Capacity Contour. To cast a complete view of how the system capacity relates to the geometry of the terminals and to provide engineering guidelines for choosing the optimal relay location, we plot in Figure 6 the capacity contour for DF when the relay is at different positions. To ease analysis, the source and the destination are placed at positions $(0,0)$ and $(0,1)$ with a normalized distance of 1 . We take the case when the signal follows the cubic law attenuation $(\delta=3)$ and $E_{s}=1$. It is interesting to observe that the contour curves are completed by two sets of arcs, cocentered at the source and the destination terminals, respectively. These arcs correspond to the capacities of the two cut sets around the source and the destination. We see that the capacity is maximized by choosing a relay that sits at the $4: 6$ section between the source and the destination. The contour is denser at the destination side than at the source side. When the relay moves farther beyond the destination, the capacity of the home channel will have exceeded that of the interuser channel, that is, $C_{S R}<C_{S D}$. Hence the relay node will stop message forwarding, and the cooperative system degenerates to a single-channel system with a capacity of $C_{S D}$.

The relation between the cooperative systems, that is, virtual antenna arrays, with the true multiantenna MIMO systems, has been the interest for a while. Work of Kramer et al. [7] and Xie and Kumar [12] reveals that decode-forward is akin to multiantenna transmission, and compress-forward (CF) is akin to multiantenna reception. It has further been suggested that DF will achieve the maximal capacity when the relay moves toward the source, and CF will achieve the maximal capacity when the relay moves toward the destination. Our results about DF are quite consistent with the multiantenna interpretation. However, using practical signal attenuation models, we have found that the optimal

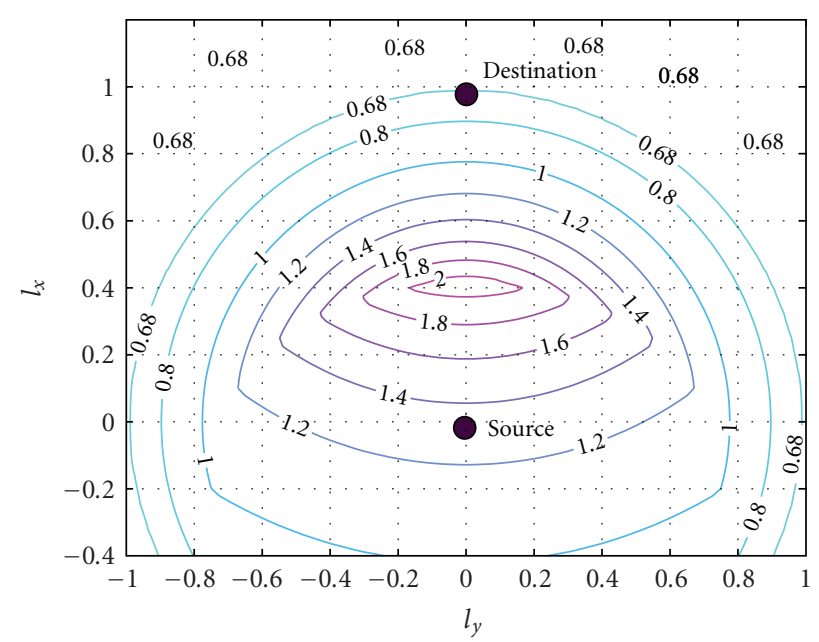

FIgURE 6: Ergodic capacity versus the location of the relay.

location for the relay needs not to be extremely close to the source.

To see why the DF relay system does not perform nearly as well as a 1-by-2 single-input multioutput (SIMO) system even when the relay gets very close to the destination (i.e., making the relay-destination channel near-perfect), consider the difference in the decoding strategies. In the SIMO system, the signals received by the multiple antennas are optimally combined and jointly decoded; whereas in the DF relay system, the signals received by the virtual antenna array are separately decoded (i.e., the relay demodulates and decodes its received signals and passes hard-decisions to the destination). In this sense, compression-forward appears to be the dual of decode-forward. If the compression of the received (analog) signals at the relay is near-lossless, then the destination will attain undistorted copies of all the signals received at the virtual antenna array and can therefore perform optimal combining and joint decoding.

\section{Relay Selection}

In previous sections, we have calculated the ergodic capacity and outage probability when the relay is at different locations. What is the expected capacity of a single-relay system whose relay is moving in a given region around the source and the destination? If there are many relay candidate nodes randomly distributed in the region and a selective singlerelay system that chooses the candidate node with the best location as its relay, what is the performance of this system? We assume that the system knows the position of each node by using GPS technology or a localization algorithm.

Assume that the distribution of the relay's location takes a certain probability distribution function $p_{X, Y}(x, y)$, where $x, y$ are coordinates of the relay's location. Let us rewrite the ergodic capacity and outage probability of DF at different $x, y$ as functions of $(x, y)$

$$
\begin{gathered}
C_{\mathrm{erg}}^{\mathrm{DF}}=C_{\mathrm{erg}}(x, y), \\
P_{\text {out }}^{\mathrm{DF}}(\theta)=P_{\text {out }}(x, y, \theta) .
\end{gathered}
$$


Let $F_{C}(c)$ and $F_{P}(p, \theta)$ denote the cumulative distribution function of the ergodic capacity and the outage probability at threshold $\theta$ of the region based on location statistics, respectively,

$$
\begin{aligned}
F_{C}(c) & =\iint_{x, y} C_{\text {erg }}(x, y) \mathrm{d} x \mathrm{~d} y, \\
F_{p}(p, \theta) & =\iint_{x, y} P_{\text {out }}(x, y, \theta) \mathrm{d} x \mathrm{~d} y .
\end{aligned}
$$

Now assume that we have $K$ candidate nodes with independent identical distribution in that region. We also assume the system can find locations of relays through some pilot signals. The system selects the candidate node with the best performance to be the relay node, while others keep silent. In other words, the system selects the node with the lowest outage probability or the highest ergodic capacity. The cumulative distribution function of this system's ergodic capacity is

$$
\begin{aligned}
F_{C, \text { sel }}(c) & =P\left(\max \left\{C_{\mathrm{erg}, i}^{\mathrm{DF}} \mid i=1,2, \ldots, K\right\}<c\right) \\
& =F_{C}(c)^{K},
\end{aligned}
$$

where $C_{\mathrm{erg}, i}^{\mathrm{DF}}$ is the ergodic capacity when the $i$ th node behaves as a relay.

The cumulative distribution function of the system outage probability is

$$
\begin{aligned}
F_{P, \text { sel }}(p, \theta) & =P\left(\min \left\{P_{\text {out }, i}^{\mathrm{DF}}(\theta) \mid i=1,2, \ldots, K\right\}<p\right) \\
& =1-P\left(\min \left\{P_{\text {out }, i}^{\mathrm{DF}}(\theta) \mid i=1,2, \ldots, K\right\}>p\right) \\
& =1-\left(1-F_{P}(p, \theta)\right)^{K}
\end{aligned}
$$

where $P_{\mathrm{out}, i}^{\mathrm{DF}}(\theta)$ is the outage probability when the $i$ th node works as a relay.

Because it is difficult to get a closed form expression of $C_{\text {region }}^{\mathrm{DF}}$ and $P_{\text {region }}^{\mathrm{DF}}(\theta)$, we use a discretized numerical method to calculate it.

(1) Divide the total region into tiny grids $(\Delta)$ of equal area. Assume that the system performance (either capacity or outage) remains invariant when the relay moves within a grid.

(2) Calculate the ergodic capacity $C_{\Delta, i}^{\mathrm{DF}}$ and the outage probability $P_{\Delta, i}^{\mathrm{DF}}(\theta)$ for each outage threshold $\theta$ in each grid (where $i$ is the index of the grid).

(3) Divide the range of the capacity or outage values into many equally spaced bins (i.e., uniform quantization of the capacity/outage values), and count the numbers of $C_{\Delta, i}^{\mathrm{DF}}$ and $P_{\Delta, i}^{\mathrm{DF}}(\theta)$ falling into each bin to form the respective histograms.

(4) Properly normalize these histograms. These normalized histograms, denoted as $\hat{p}_{C}(c)$ and $\hat{p}_{P}(p, \theta)$, serve as the discretized approximations of the capacity distribution and outage distribution (which are functions of the location distribution of the relay).

When both the area of the grid and the step size of the bin approach zero, $\hat{p}_{C}(c)$ and $\hat{p}_{P}(p, \theta)$ approach the probability distribution function of the ergodic capacity $p_{C}(c)$ and the outage probability $p_{P}(p, \theta)$ of the region, respectively. From $\hat{p}_{C}(c)$ and $\hat{p}_{P}(p)$, we can compute the respective cumulative distribution functions (cdf), $\widehat{F}_{C}(c)$, and $\hat{F}_{P}(p, \theta)$, which are used as approximation to the true cdf's $F_{C}(c)$ and $F_{P}(p)$.

As an example, we consider the case when candidate relay nodes are confined to a square region around the source and the destination. Following the setup in Figure 2, we place the source and the destination at locations $\left(l_{x}, l_{y}\right)=(0,0)$ and $(1,0)$, respectively, and choose both the length and the width of the relay region to be unity: $l_{x} \in[0,1]$ and $l_{y} \in[-0.5,0.5]$. Without loss of generality, assume that the candidate relay nodes are all independent and moves around in the region uniformly and randomly.

When no candidate relay node is available, the system degrades into a noncooperative system, and the source keeps transmitting different messages in both time slots. When there are more than one candidate nodes, the system becomes a best selective single-relay system, where a message is transmitted by the source in the first time slot and relayed by the best candidate (i.e., in the best location) in the second time slot.

In Figure 7, solid curves represent the average ergodic capacity of the best selective single-relay system with the number of candidates from 0 to 40 , where the average is taken among all the possible relay locations. In the figure, when the pass loss is relatively small, that is, $\delta=2,3$, the availability of only one or two candidate nodes may not provide any gain in terms of the average ergodic capacity. As the number of candidate nodes increases, the benefit of user cooperation begins to outweigh the loss in bandwidth efficiency caused by cooperation. For the case of $\delta=4$, because of the severe path loss, the diversity gain provided by the relay system becomes crucial, and the relay system appears to unanimously outperform the noncooperative system even though there is only one candidate to select from. As the number of the candidates increases without bound, the average system capacity approaches a limit, which is achieved by positioning (at least) one relay candidate at the optimal location at all times.

Figure 8 demonstrates the average outage probability of the best selective single-selection system (solid curves). The performances are similar to the case of the capacity, except that, regardless of what value of $\delta$ or how many candidate nodes are used, cooperation always elevates the outage performance. Further, the outage probability drops at a faster rate than the increase in the capacity (Figure 7) as the size of the candidate pool increases. As soon as the size of the candidate pool reaches 3 to 5, the outage performance get quite close to the limit (dashed lines). This suggests that, in the best selective single-relay system, it is sufficient for the system to keep track of only 3 to 5 candidates in order to reap most of the gain promised by the theory. 


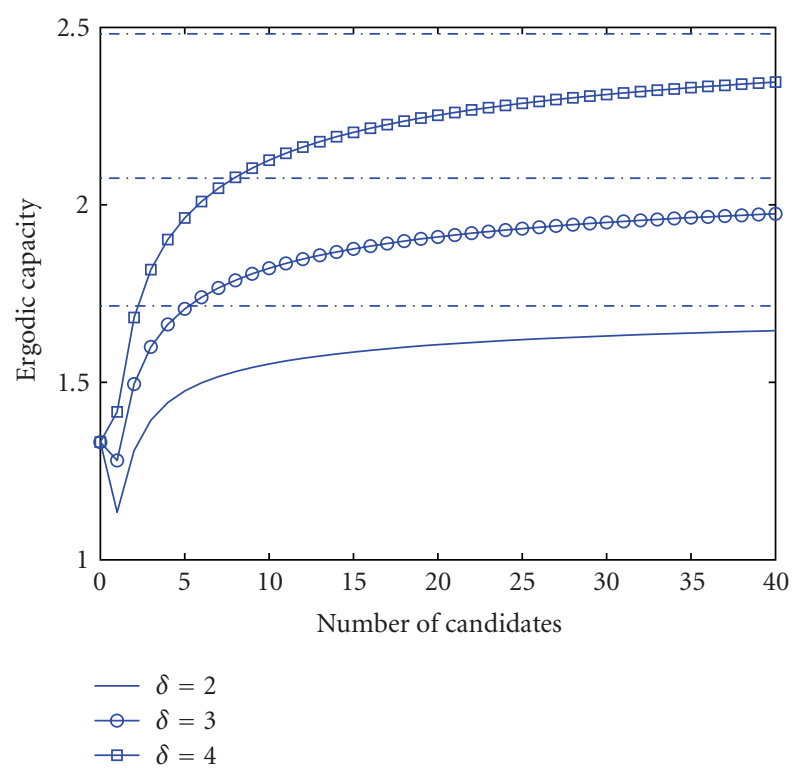

FIGURE 7: Ergodic capacity of single-relay selection system with up to 40 candidate nodes.

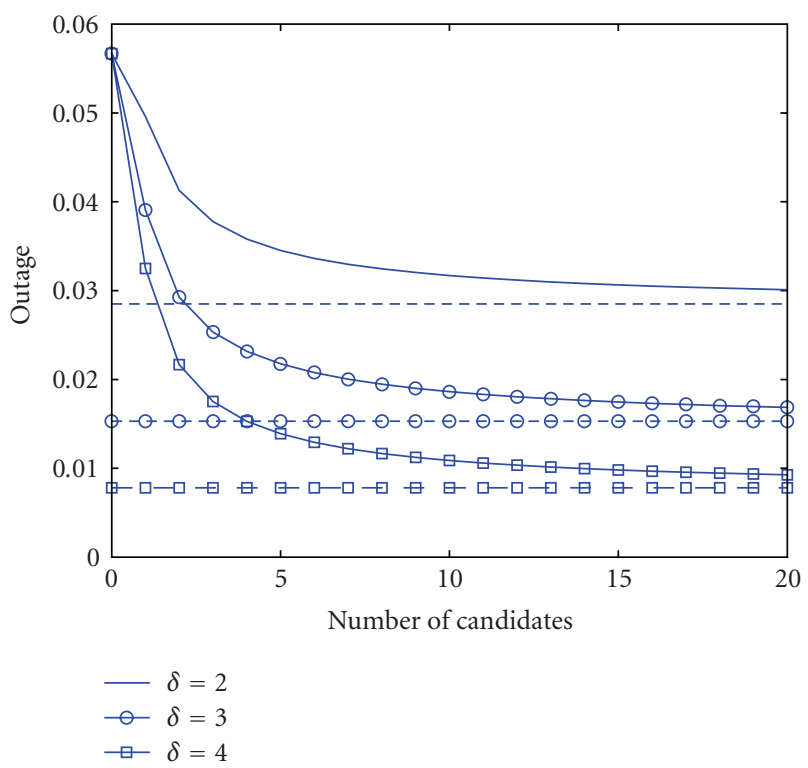

Figure 8: Outage of single-relay selection system with up to 20 candidate nodes.

\section{Conclusion}

We have analyzed the performances of amplify-forward and decode-forward, the two basic signal relaying modes, for a single-relay system in Rayleigh fading environment. The max-flow min-cut theory is used as the base approach, and the performance measure is quantified by the ergodic capacity and the outage probability. We have explicitly taken into account the geometry of the nodes, the distances between them, and the resulting attenuation of radio signals and weighted the information as a function of the transmission distances.
In the case of amplify-forward, we have demonstrated an interesting symmetry property in both the capacity and the outage results. We have shown that the maximum value is achieved when the relay sits on the median line between the source and the destination in most cases, with the exception when the power is very low (below a certain threshold). In the latter case, two symmetric optimal locations on either side of the median line are observed, but our numerical evaluation shows that for the relay to locate anywhere between these two optimal locations incurs only a small performance degradation. Hence, the median point remains a convenient and good choice.

In the case of decode-forward, our capacity and outage results confirm that the system operates much like a multiantenna transmission system [12]. Using practical signal attenuation models and the max-flow min-cut theorem, we have found that the optimal relay location is somewhere around, but not extremely close to, the source. To provide a complete picture of the system performance as a function of the geometry, a capacity contour plot is computed for the DF system. We note that a similarly contour plot can also be computed for the case of AF, but the computational complexity is much higher. (Each point for AF requires a 3-dimensional integration for the case of AF, but only a 2dimensional integration for the case of DF.) Further, the contour plot would be more useful for DF than for AF, since the symmetry condition of AF and the "median point rule" make it easier to choose the relay location.

Following this geometry-inclusive analysis, a best selective single-relay system is proposed and analyzed. We consider the case where multiple candidates may be available, but only the one at the best location will be chosen each time to relay the message. Using a discretized numerical method, we have demonstrated the average system capacity and outage as a function of the size of the candidate pool. We observe that when the pass loss is relatively small, the availability of only one candidate node may not render an average system capacity higher than a noncooperative system (due to the loss in bandwidth efficiency). When more candidate nodes are available or when the pass loss is severe, the system performance of the relay system quickly picks up and soon outperforms that of the noncooperative system. We have further demonstrated that the source needs only keep track of some 3 to 5 candidate relays in order to harness most of the "geometric" benefits available to a selective relay system.

\section{Acknowledgments}

The work is supported in part by the National Science Foundation under Grant no. CCF-0430634 and by the Commonwealth of Pennsylvania, Department of Community and Economic Development, through the Pennsylvania Infrastructure Technology Alliance (PITA).

\section{References}

[1] A. Sendonaris, E. Erkip, and B. Aazhang, "User cooperation diversity_part I: system description," IEEE Transactions on Communications, vol. 51, no. 11, pp. 1927-1938, 2003. 
[2] J. N. Laneman, D. N. C. Tse, and G. W. Wornell, "Cooperative diversity in wireless networks: efficient protocols and outage behavior," IEEE Transactions on Information Theory, vol. 50, no. 12, pp. 3062-3080, 2004.

[3] M. Janani, A. Hedayat, T. E. Hunter, and A. Nosratinia, "Coded cooperation in wireless communications: space-time transmission and iterative decoding," IEEE Transactions on Signal Processing, vol. 52, no. 2, pp. 362-371, 2004.

[4] R. U. Nabar, H. Bolcskei, and F. W. Kneubuhler, "Fading relay channels: performance limits and space-time signal design," IEEE Journal on Selected Areas in Communications, vol. 22, no. 6, pp. 1099-1109, 2004.

[5] M. Yu and J. Li, "Is amplify-and-forward practically better than decode-and-forward or vice versa?" in Proceedings of the IEEE International Conference on Acoustics, Speech, and Signal Processing (ICASSP '05), vol. 3, pp. 365-368, Philadelphia, Pa, USA, March 2005.

[6] Z. Lin, E. Erkip, and A. Stefanov, "Cooperative regions for coded cooperative systems," in Proceedings of the IEEE Global Telecommunications Conference (GLOBECOM '04), vol. 1, pp. 21-25, Dallas, Tex, USA, November-December 2004.

[7] G. Kramer, M. Gastpar, and P. Gupta, "Cooperative strategies and capacity theorems for relay networks," IEEE Transactions on Information Theory, vol. 51, no. 9, pp. 3037-3063, 2005.

[8] B. Zhao and M. C. Valenti, "Practical relay networks: a generalization of hybrid-ARQ," IEEE Journal on Selected Areas in Communications, vol. 23, no. 1, pp. 7-18, 2005.

[9] T. Cover and A. El Gamal, "Capacity theorems for the relay channel," IEEE Transactions on Information Theory, vol. 25, no. 5, pp. 572-584, 1979.

[10] M. A. Khojastepour, A. Sabharwal, and B. Aazhang, "Lower bounds on the capacity of Gaussian relay channel," in Proceedings of the 38th Annual Conference on Information Sciences and Systems (CISS '04), pp. 597-602, Princeton, NJ, USA, March 2004.

[11] A. Host-Madsen and J. Zhang, "Capacity bounds and power allocation for wireless relay channels," IEEE Transactions on Information Theory, vol. 51, no. 6, pp. 2020-2040, 2005.

[12] L.-L. Xie and P. R. Kumar, "An achievable rate for the multiple level relay channel," IEEE Transactions on Information Theory, vol. 51, no. 4, pp. 1348-1358, 2005. 

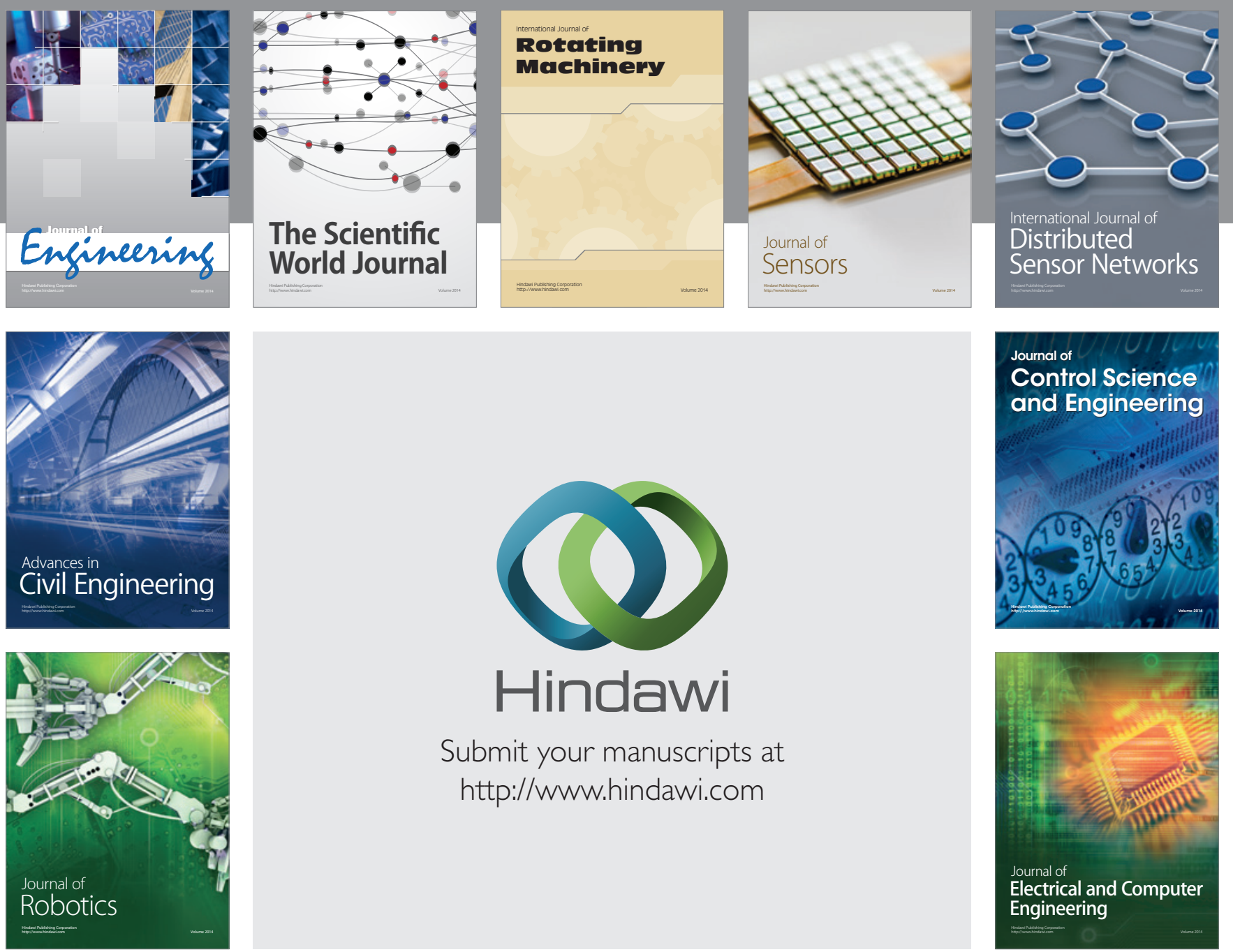

Submit your manuscripts at

http://www.hindawi.com
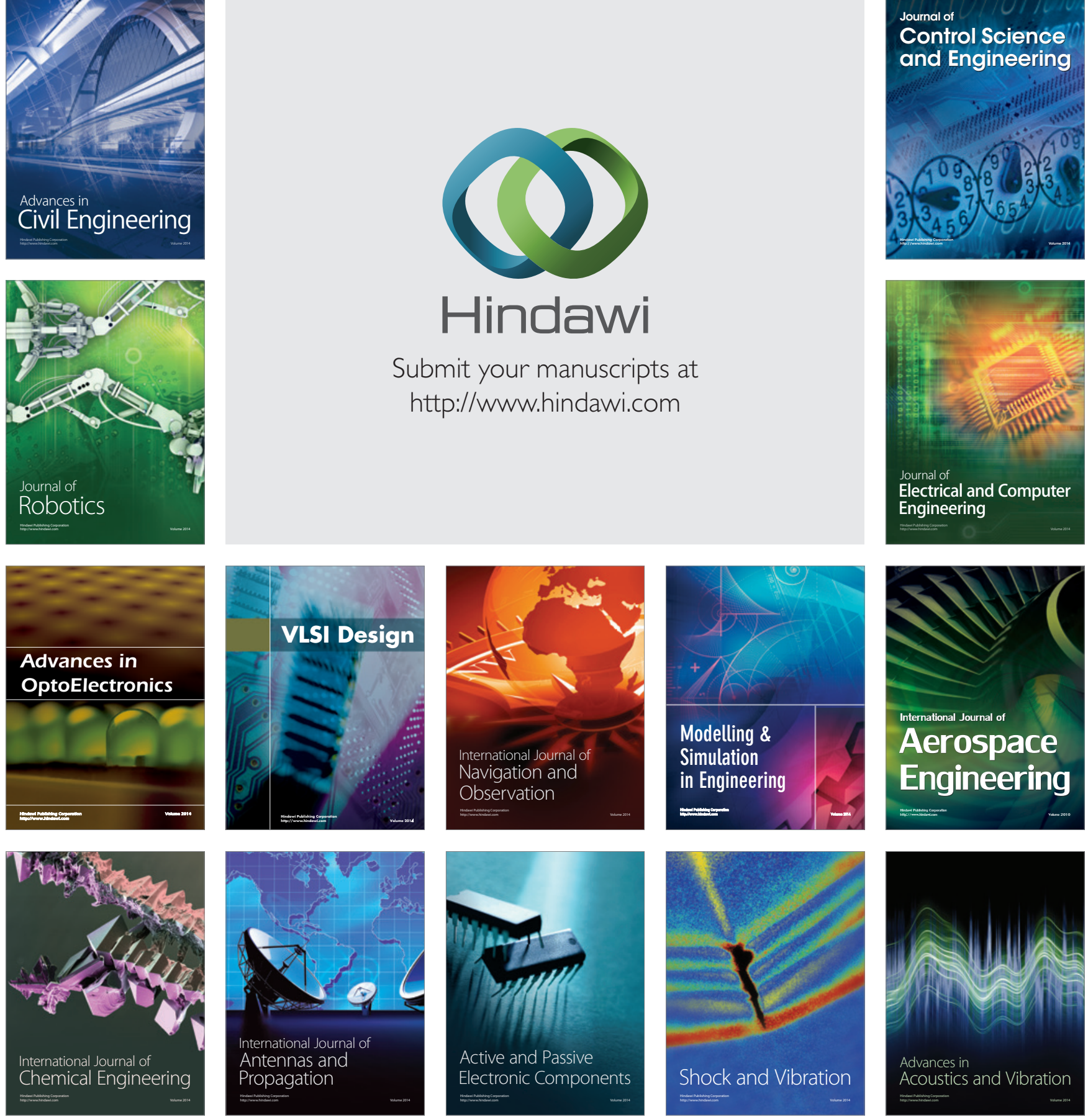